\title{
Zum Gerichtssystem in Libyen
}

\author{
Von Hans-Georg Ebert
}

Analysen und Berichte zum libyschen Rechts- und Justizsystem - an sich schon eher eine Seltenheit außerhalb der libyschen Grenzen - richten die Aufmerksamkeit des Interessenten vor allem auf Spektakuläres. Tatsächliche und vermeintliche dubiose Gerichtspraktiken vermischen sich zu einem fest zementierten Vorurteil, wonach es in Libyen jenseits von Lynchjustiz und gerichtlichem Chaos keinerlei gesetzmäßigen Prinzipien gebe. Todesurteile und öffentliche Vollstreckung gehörten in der Vergangenheit durchaus zum Instrumentarium libyscher Politik, so z.B. im Zuge der innenpolitischen Ereignisse am 8.5.1984, als sich Basisvolkskongresse - offiziell ermuntert - aufschwangen, angebliche Feinde des Landes hinzurichten. 1 Unabhängig davon, ob die Verurteilten die ihnen zur Last gelegten Verbrechen tatsächlich in vollem Ausmaß begangen hatten oder nicht, wiesen diese Verfahren doch erhebliche formale und sachliche Mängel auf, wurden fixierte gesetzliche Grundlagen mißachtet. Gegenstand berechtigter Kritik ist ferner die sog. "islamische Gesetzgebung" aus den Jahren 1971 bis 1974, die bestimmte Normen der saria kodifiziert.2 Mit Ausnahme von Auspeitschungen bei Alkoholkonsum wurden jedoch die im Koran festgelegten sog. hudud-Strafen kaum verhängt. ${ }^{3}$ Im "Grünen Buch" M. al-Quaddafis heißt es u.a.: "In der Religion werden die meisten materiellen Strafen bis auf den Tag des Jüngsten Gerichts zurückgestellt. Der größte Teil ihrer Regeln besteht in Ermahnungen, Anleitungen und Antworten auf Fragen. Dieses Gesetz zeigt den angebrachten Respekt vor dem Menschen. Die Religion erkennt keine irdischen Strafen an, außer in extremen Fällen, in denen sie zum Schutz der Gesellschaft notwendig sind."4 Das am 12.6.1988 verabschiedete "Große Grüne Dokument für den Frieden und die Menschenrechte im Zeitalter der Massen"

1 Vgl.z.B. ein Urteil des Basisvolkskongresses in Nalut-Zentrum. Darin werden zwei Personen zum Tode verurteilt wegen "Mitgliedschaft in der Organisation der Muslimbrüder und Ausübung von Sabotageakten gegen die Volksmacht". In: Az-zahf al-ahdar, Tarabulus 4.6.1984, S. 1.

2 Diese Gesetze sind aufgeführt in: Qanun al-waqf fi al-gumhuriya al-arabiya al-libiya. Bairut 1973, S. 77. Weiterhin sind die entsprechenden Gesetzestexte enthalten in: Qanun az-zakat fi algurnhuriya al-arabiya al-libiya. Tarabulus 1972 (hier sind auch die Durchführungsverordnungen daza veröffentlicht), Qanun haddai as-sariqa wa al-hiraba fi al-gumhuriya al-arabiya al-libiya. Tarabulus 1972. Besonders der ägyptische Jurist A.A. Mansur hat einen entscheidenden Anteil an der Ausarbeitung dieser Gesetze. Vgl. seine Arbeit: Mansur, A.A., Hutwa ra'ida nahwa tatbiq ahkam as-saria al-islamiya fi al-gumhuriya al-arabiya al-libiya. Tarabulus, Bairut 1972.

3 Badry, R., Die Entwicklung der Dritten Universaltheorie (DUT) Muammar al-Qaddafis, in: Theorie und Praxis aus ideengeschichtlicher und historischer Sicht, Frankfurt a.M., Bem, New York 1986, S. 199.

4 Al-Kadhafi, M., Das Grüne Buch. Die Dritte Universaltheorie. Kapitel 1-3. Bonn 1980, S. 32-33. 
verkündet: "Die Jamahiriya ${ }^{5}$ garantiert jedem das Recht, Gerechtigkeit zu suchen; sie sichert die Unabhängigkeit der Justiz und das Recht jedes einzelnen auf einen fairen und ehrlichen Prozeß."6

Die Untersuchung von Struktur und Mechanismus des Gerichtssystems in Libyen erfordert zwei grundsätzliche Vorbemerkungen:

1. Es geht hierbei nicht primär um eine politische Wertung von rechtlichen Instituten und Institutionen, sondern um die Darstellung des vom Gesetzgeber beabsichtigten Aufbaus der Justiz.

2. Die Gesetze und Beschlüsse zum libyschen Gerichtssystem sind aufgrund ihrer relativ schnellen Emeuerung und Ergänzung nur schwer überschaubar.7 Die Entwicklung einer stabilen und funktionierenden Justiz verkompliziert sich auf diese Weise beträchtlich. Gleichzeitig wird die Rechtssicherheit der Bürger in Libyen erheblich eingeschränkt.

Generell zeichnet für die Organisation und die materiell-technische Absicherung des Gerichtssystems in Libyen das Sekretariat (Ministerium) für Justiz verantwortlich. Von 1986 bis 1989 wurden zwar dessen Kompetenzen auf die Bezirke (Baladiyas) übertragen, gleichzeitig jedoch per Beschluß des Allgemeinen Volkskomitees Nr. 520 von 1986 ein "Allgemeines Gremium für Justizangelegenheiten" (al-hai'a al-amma li-su'un al-qada')8 geschaffen, welches gemäß Art. 3 faktisch die Aufgaben des Justizsekretariats wahrzunehmen hatte. Lediglich die jenigen Rechte und Pflichten des Justizsekretariats, die Fragen der inneren Sicherheit betrafen, fielen laut Beschluß des Allgemeinen Volkskomitees Nr. 522 von 19869 in die Verantwortlichkeit der entsprechenden Sekretariate auf Baladiya-Ebene. Somit läßt sich eine Kontinuität des libyschen Gerichtssystems hinsichtlich seiner zentralen Organisationsstruktur und seines pyramidenförmigen Aufbaus konstatieren. Es basiert in erster Linie auf dem "Gesetz über das Gerichtssystem Nr. 51 von 1976".10 Dieses Gesetz folgt in wesentlichen Aussagen seinem Vorläufer Nr. 29 von 1962.11 Die Zuständigkeit der libyschen Gerichte erstreckt sich auf Straf- und Zivilsachen, Verwaltungsangelegenheiten und - wie an anderer Stelle dieser Arbeit ausführlicher erläutert - auf Personenstandsfra-

5 Offizielle Staatsbezeichnung Libyens; bedeutet etwa "Staat der Massen".

6 Archiv der Gegenwart, St. Augustin 12.6.1988, S. 32258.

7 Vgl. Ebert, H.-G., Gesetz und Gesetzgebung in Libyen, in: Staat und Recht (Potsdam-Babelsberg) 38 (1989) 5, S. 404.

8 Al-garida ar-rasmiya. Tarabulus 28.10.1986, Nr. 21, S. 764-770.

9 Al-farida ar-rasmiya. Tarabulus 28.10.1986, Nr. 21, S. 771-773; vgl. insbesondere Art. 1.

10 Qanun nizam al-qada' raqm 51 li-sana 1976 (Auszug aus: Al-garida ar-rasmiya Nr. 45 von 1976).

11 Vgl. dazu die ausführliche Interpretation in: Gira, A. al-M: A. al-A., At-tanzim al-qada'i fi libiya. Bingazi 1978. Im folgenden sollen die grundsätzlichen Ausführungen dieses Buches Berücksichtigung finden. 
gen. ${ }^{12}$ Im allgemeinen ist das libysche Gerichtssystem formal auf folgende generelle Prinzipien verpflichtet:

a) die Unabhängigkeit des Gerichtswesens (Trennung der Gewalten);

b) die Gleichheit vor dem Gericht;

c) die Gebührenpflicht der Gerichtsverfahren;

d) die Abstufung der Gerichte mit umrissenen Kompetenzen;

e) das System des Einzelrichters (bei den sog. Bagatellgerichten und z.T. bei den Grundgerichten) und das mehrerer Richter gleichzeitig13;

f) die Offentlichkeit der Sitzungen, die nur in gesetzlich niedergelegten Fällen aufgehoben werden kann, und

g) die Durchfürung von direkten mündlichen Gerichtsverfahren (Gerichtssprache ist Arabisch) bei Anerkennung schriftlicher Dokumente.14

Die unterste Stufe des Gerichtssystems in Libyen bilden die Bagatellgerichte (al-mahakim al-guz'iya; Art. 7-13 des Gesetzes Nr. 51 von 1976). Sie sind sowohl für Zivilangelegenheiten mit geringem Streitwert als auch für Strafsachen zuständig. Letztere beziehen sich auf Vergehen und Verfehlungen (gunah wa muhalafat), weswegen diese Gerichte auch als "Gerichte für Vergehen und Verfehlungen" bezeichnet werden. Einige Urteile dieser Gerichte erscheinen in Kurzfassung in den Zeitungen des Landes, so in der "Al-fagar algadid".15 Den Bagatellgerichten entsprechen rangmäßig die sog. "Abgelegenen Gerichte" (al-mahakim an-na'iya) in Wüstendörfern und -städten (Art. 10-13 des Gesetzes Nr. 51 von 1976). Auf nächst höherer Ebene - den Grundgerichten (al-mahakim al-ibtida'iya; Art. 6 des Gesetzes Nr. 51 von 1976) - kann zum einen in erster Instanz und zum anderen in zweiter Instanz (Appellationen bezüglich von Entscheidungen der Bagatellgerichte) verhandelt werden. Im Zuständigkeitsbereich eines Grundgerichts liegen im allgemeinen mehrere Bagatellgerichte. Angelegenheiten des Personenstandsrechts, des Erbrechts und des Waqf-Rechts (Recht der Religiösen Stiftungen) - diese Rechtszweige wurzeln in starkem Maße im traditionellen islamischen Recht (saria) - fallen auch in die Kompetenz der Grundgerichte, was zuvor im "Gesetz über die Maßnahmen der islamischen Gerichte (almahakim as-sariya) vom 15.11.1958" sowie im "Gesetz Nr. 87 von 1973 über die Vereinigung der Judikative" (d.h. der Zivil- und saria-Gerichtshöfe) verankert war.16 Nach den Ubergangsbestimmungen des Gesetzes Nr. 51 von 1976 sollen aber "islamische Gerichte

12 Vgl. folgende Gesetze: "Gesetz über Strafen und Strafmaßnahmen", "Gesetz über Zivil- und Handelsverfahren", "Gesetz über die Verwaltungsgerichtsbarkeit Nr. 88 von 1971" sowie "Gesetz Nr. 10 vom 1984 über die Bestimmungen der Eheschließung und Scheidung sowie deren Wirkungen".

13 Vgl. Art. 3-13 des Gesetzes Nr. 51 von 1976.

14 Vgl. Gira, A. al-M. A al-A., At-tanzim..., a.a.O., S. 35-52 und Ar. 20-23 des Gesetzes Nr. 51 von 1976.

15 Z.B. al-fagar al-gadid. Tarabulus 29.5.1984, S. 6 und ebenda 19.5.1984, S. 6.

16 Gira, A. al-M.A al-A., At-tanzim..., a.a.O., S. 61. 
bis zur Veröffentlichung des Personenstandsgesetzes" in bestimmten, von der saria geregelten Angelegenheiten (Art. 160) weiter existieren. Daraus resultiert eine widersprüchliche judikative Situation: Ein Teil der Sachgebiete des Art. 160 ist bereits im "Gesetz über die Bestimmungen der Eheschließung und Scheidung sowie deren Wirkungen Nr. 10 von 1984" enthalten, ein anderer Teil muß noch entsprechend dem malikitischen Ritus vor saria-Gerichten zur Verhandlung gelangen.17

Die Appellationsgerichte (mahakim al-isti'naf; Art. 3-5 des Gesetzes 51 von 1976), welche in mehrere Kammem gegliedert sind18, befassen sich mit schwerwiegenden Fällen, die die verfügten Urteile widerspiegeln.19 Sie können in erster Instanz und in zweiter Instanz verhandeln. Gemäß "Gesetz Nr. 88 von 1971 über die Verwaltungsgerichtsbarkeit" haben die Appellationsgerichte bei Verwaltungsstreitigkeiten in erster Instanz zu tagen. Die Appellationsgerichte für Strafsachen werden Kriminalgerichte (mahakim al-gunayat) genannt (Art. 4 des Gesetzes Nr. 51 von 1976). In jeder Stadt, die über ein Grundgericht verfügt, kann ein solches Kriminalgericht wirken.20

Das Oberste Gericht (al-mahkama al-ulya) gilt als das höchste rechtsprechende Organ in Libyen. Seine Bildung, Kompetenzen und Arbeitsweise ergeben sich aus dem "Gesetz über die Reorganisation des Obersten Gerichts Nr. 6 von 1982".21 Dieser Rechtsakt ersetzt das bislang gültige "Gesetz über das Oberste Gericht vom 10.11.1953" sowie das entsprechende Innere Statut. Das Oberste Gericht besteht gemäß Art. 1 aus seinem Vorsitzenden (ra'is) und einer "genügenden Anzahl von Räten" (adad kaf min al-mustasarin). Es ist in mehrere Kammern unterteilt (Art. 2). Sitz des Gerichts ist Tripolis (Art. 4). Die Emennung der Räte, deren geforderte Eigenschaften in Art. 5 verankert sind, und des Vorsitzenden erfolgt auf Beschluß des Allgemeinen Volkskongresses (Art. 6). Art. 15 bis Art. 22 enthalten die Modalitäten einer sog. "Veto-Anwaltschaft" (niyaba an-naqd), deren Aufgabe darin besteht, Rechtsurteile bezüglich der an das Oberste Gericht herangetragenen Appellationsklagen zu erarbeiten (Art. 17). Die vom Obersten Gericht erlassenen Anordnungen und Entscheidungen in strafrechtlichen Appellationen werden auf Befehl der "Veto-Anwaltschaft" in Ubereinstimmung mit dem "Gesetz über die Strafmaßnahmen" durchgesetzt (Art. 18). Im "Beschluß des Allgemeinen Volkskomitees Nr. 708 von 1982 über die Organisation der

17 Ebert, H.-G., Die Interdependenz von Staat, Verfassung und Islam im Nahen und Mittleren Osten in der Gegenwart. Leipzig 1989, Diss. B., S. 53-54.

18 Art. 4 und 5 des Gesetzes Nr. 51 von 1976: Kammem für Strafsachen und für Verwaltungsgerichtsbarkeit.

19 Z.B. beschließen die Appellationsgerichte drastische Strafmaßnahmen wie lebenslänglichen Freiheitsentzug und Aberkennung der Zivilrechte. Vgl. al-fagar al-gadid. Tarabulus 21.4.1984, S. 6.

20 Hatama, M.N., Sarh al-igra'at al-guna'iya fi al-qanun al-libi. Bingazi 1980, S. 298-301.

21 Das Gesetz enthält insgesamt 57 Artikel. Daran schließt sich ein kurzer Anhang an. Al-garida arrasmiya. Tarabulus 7.8 .1982 , Nr. 22, S. 754-768. 
Veto-Anwaltschaft"22 erscheint letztere Funktion in Art. 2, wobei die "Angestellten des Gerichtsvollzuges" (ma'muru ad-dabt al-qada'i) 23 die Anweisungen der "Veto-Anwaltschaft" auszufuhren haben. Laut Art. 1 des Beschlusses Nr. 708 von 1982 werden die Mitglieder der "Veto-Anwaltschaft" als "Mitarbeiter des Gerichtswesens" (rigal al-qada') klassifiziert.24 Ein auf Beschluß des Obersten Gerichts gebildetes "Komitee zur Inspektion" (lagna li-t-taftis) überwacht die Arbeit der "Veto-Anwaltschaft" (Art. 9 des Beschlusses Nr. 708 von 1982).

Die Kompetenzen des Obersten Gerichts schreiben Art. 23 bis Art. 26 fest. In folgenden Fragen ist das Oberste Gericht als Ganzes ausschließlich zuständig (Art. 23):

1. Kompetenzstreitigkeit zwischen den Gerichten und einem Organ mit sondergerichtlicher Vollmacht (giha qada' istitna'i);

2. Streit, der aus der Durchfulhrung von zwei widersprüchlichen endgültigen Entscheidungen resultiert - die eine von einem Gericht, die andere von einem Organ mit sondergerichtlicher Vollmacht, und

3. Weiterleitung einer Klage an die Allgemeine Körperschaft (al-gamiya al-umumiya) durch eine Kammer des Gerichts, um ein Gesetzesprinzip aus vorangegangenen Entscheidungen zu verwerfen.

Gemäß Art. 24 verfügt das Oberste Gericht als "Vetogericht" bezüglich von Berufungsklagen in folgenden Fragen über Kompetenzen:

1. Entscheidungen in Zivil-, Handels- und Personenstandsangelegenheiten in Übereinstimmung mit dem "Gesetz über Zivil- und Handelsverfahren";

2. Entscheidungen der Appellationsgerichte, die als Gremium der Verwaltungsgerichtsbarkeit (hai'a qada' idari) tagen, gemäß Gesetz Nr. 88 von 1971 sowie

3. Entscheidungen in Strafangelegenheiten gemäß "Gesetz über die Strafmaßnahmen".

Ferner kann das Oberste Gericht in Strafsachen eingreifen, die Verbüßung einer Strafe durch Beschluß beenden (Art. 25).25 Alle Gesetzesprinzipien, die das Oberste Gericht in seinen Beschlüssen verkündet, müssen von sämtlichen Gerichten und anderen Organen in

22 Beschluß in: Al-garida ar-rasmiya. Tarabulus 31.1.1983, Nr. 3, S. 105-114. Insgesamt umfaßt dieser Beschluß 38 Artikel.

23 Vgl. dazu die Beschlüsse 1422 von 1981 und Nr. 238 von 1982 des Allgemeinen Volkskomitees. $\mathrm{Zu}$ den "Angestellten des Gerichtsvollzuges" zählen auch die Mitarbeiter des Zolls, der BaladiyaGarde und der Polizei. Gemäß Beschluß Nr. 87 von 1979 des Sekretärs des Allgemeinen Volkskomitees für Kommunikation und Seetransport werden die Mitglieder der Volkskomitees für Kommunikation und Seetransport ebenso als "Angestellte des Gerichtsvollzuges" betrachtet (Art. 2). In: Al-garida ar-rasmiya. Tarabulus 5.2.1981, Nr. 4, S. 115-116.

24 Vgl. auch Art. 21 des "Gesetzes über die Reorganisation des Obersten Gerichts Nr. 6 von 1982".

25 Eine solche Entscheidung kann auch durch Beschluß des Allgemeinen Volkskomitees erfolgen. Vgl. z.B. Beschluß Nr. 128 von 1984 in: Al-garida ar-rasmiya. Tarabulus 29.3.1984, Nr. 8, S. 334. 
Libyen respektiert werden (Art. 31). Ein "Technisches Büro" (maktab fanni) organisiert die regelmäßige Herausgabe der Beschlüsse des Obersten Gerichts. Die entsprechende Zeitschrift (magalla al-mahkama al-ulya) erscheint viermal jährlich und ist nach Sachgebieten geordnet: Personenstands-, Verwaltungs-, Zivil- Strafberufungsklagen u.a.26

Das Oberste Gericht verfügt über vielfältige rechtliche Möglichkeiten, Urteile zu bestätigen, zu revidieren oder auszusetzen. Uberdies kontrolliert es seit 1988 den Muftirat (dar alifta') direkt. Dieses Gremium erteilt auf Anfrage Rechtsgutachten hinsichtlich der saria und erläßt Mitteilungen zu ihrer Anwendung (z.B. Erbrecht). Somit sichert das Oberste Gericht wesentlich die Einflußnahme der staatlich Herrschenden auf die Rechtsprechung und garantiert eine weitgehende Gleichschaltung der Judikative mit dem Regime.

Die "Mitarbeiter des Gerichtswesens und der Staatsanwaltschaft" (rigal al-qada' wa anniyaba al-amma) rekrutieren sich zum einen aus den Richtern (qudat, Sg. qadi) und zum anderen aus den Staatsanwälten (ada' an-niyaba al-amma). Erstere umfassen gleichsam die Räte (mustasarun) an den Appellationsgerichten und am Obersten Gericht. Richter kann nur werden, wer die libysche Staatsangehörigkeit besitzt, über einen guten Leumund verfügt, nicht vorbestraft ist, eine rechtswissenschaftliche Qualifikation nachweisen kann und mindestens 27 (bei Räten 35) Jahre alt ist (vgl. Art. 43 des Gesetzes Nr. 51 von 1976).27 Seit April sind auch Frauen als Richter zugelassen. Im Gegensatz zu vergleichbaren Regelungen in anderen arabischen Ländern (etwa Ägypten) anerkennt der libysche Staat ein Zeugnis über das erfolgreiche Studium der saria als hinreichende fachliche Voraussetzung für ein Richteramt.

Die Funktion der Staatsanwaltschaft (an-niyaba al-amma) üben gemäß Art. 36 des "Gesetzes über das Gerichtssystem Nr. 51 von 1976"28 die Vorsitzenden der Anwaltschaft (ru'asa' an-niyaba) aus. Ihnen stehen zur Seite:

- Vertreter der Anwaltschaft (nuwab an-niyaba);

- Prozeßvertreter (wukala');

- Assistenten (musaidun) und

- Gehilfen (muawinun).

Jedes Gericht beschäftigt zudem eine bestimmte Anzahl von technischen Angestellten (muwazzafu al-mahakim wa an-niyaba al-amma). Diese arbeiten als Sekretäre (kataba,

Die Zeitschrift erscheint im Januar, April, Juli und Oktober des jeweiligen Jahres und enthält sowohl eine Beschreibung der Berufungsverfahren als auch - alphabetisch geordnet - eine Übersicht über die entsprechenden gesetzlichen Prinzipien.

27 Vgl. Gira, A. al-M. A al-A., At-tanzim..., a.a.O., S. 84.

28 In der veränderten Fassung gemäß "Beschluß Nr. 8 von 1982 des Allgemeinen Volkskongresses über die Veränderung einiger Bestimmungen des Gesetzes über das Gerichtssystem". In: Al-garida ar-rasmiya. Tarabulus 25.8.1983, Nr. 25, S. 575 . 
auch umana') sowie Gerichtsdiener (muhdirun). Außerdem können verschiedene Sachverständige (hubara') zu Rate gezogen werden.

Mit dem "Gesetz Nr. 4 von 1981 über die Schaffung einer Volksrechtsanwaltschaft" und der dazugehörigen Durchführungsverordnung (la'iha tanfidiya) vom 16.5.1981 erhielten die Rechtsanwälte (muhamun) des Landes eine neue rechtliche Basis, welche sie direkt an die Gerichte bindet. Gemäß Art. 1 der Durchführungsverordnung29 besteht die "Verwaltung der Volksrechtsanwaltschaft" aus Zweigstellen (furu) an den Appellationsgerichten und Büros (makativ) an den Grundgerichten. Auf Beschluß des Allgemeinen Volkskomitees für Justiz können solche Büros auch an den Bagatellgerichten gebildet werden. Die jeweilige gerichtliche Ebene - Oberstes Gericht, Appellationsgericht, Kriminalgericht, Grundgericht, Bagatellgericht - erfordert vom Rechtsanwalt eine bestimmte festgelegte Klassifizierungsstufe (Gruppe a-c für das Oberste Gericht, Klassen 1-4 für die anderen Gerichte; Art. 22). Die "Verwaltung der Volksrechtsanwaltschaft" gliedert sich sachgebietsmäßig in vier Abteilungen: Zivilklagen, Strafklagen, Personenstandsklagen sowie Verwaltungs- und Verfassungsfragen (Art. 9).30

Seit März 1990 sind in Libyen auch wieder unabhängige Rechtsanwälte zugelassen, so daß sich der gerichtliche Beistand für die Bürger oder juristischen Personen nicht mehr auf die am jeweiligen Gericht tätigen Rechtsanwälte reduziert. Nach wie vor ist jedoch insbesondere für Nichtlibyer und nichtlibysche juristische Personen die erfolgreiche Abwicklung eines juristischen Verfahrens vor einem libyschen Gericht schwierig, obwohl bereits Art. 14 der Durchführungsverordnung in der Fassung des Beschlusses Nr. 945 von 1981 den ausländischen und internationalen Gesellschaften und Organen die Möglichkeit einräumte, bei Klagen gegen sie oder von ihnen eine Zweigstelle oder ein Büro der "Verwaltung der Volksrechtsanwaltschaft" gegen Entgelt zu kontaktieren.

Am 22.8.1982 erließ der Sekretär des Allgemeinen Volkskomitees für Justiz den "Beschluß Nr. 568 von 1982 über die Regeln und Maßnahmen der Gerichtsaufsicht" (at-taftis alqada'i). 31 In Übereinstimmung mit dem Gesetz Nr. 51 von 1976 und Art. 1 dieses Beschlusses emennt der Sekretär des Allgemeinen Volkskomitees für Justiz mit Zustim-

29 Durchführungsverordnung vom 16.5.1981 inAl-garida ar-rasmiya. Tarabulus 30.5.1981, Nr. 24, S. 713-728. Veränderte Fassung des Art. 1: "Beschluß Nr. 417 von 1982 des Allgemeinen Volkskomitees über die Veränderung einiger Bestimmungen der Durchführungsverordnung des Gesetzes Nr. 4 von 1981 über die Schaffung einer Verwaltung der Volksrechtsanwaltschaft". In: Al-garida ar-rasmiya. Tarabulus 19.10.1982, Nr. 31, S. 1087-1089.

30 In der Fassung des Beschlusses des Allgemeinen Volkskomitees für Justiz Nr. 945 von 1981 über die Veränderung einiger Bestimmungen der Durchfühnungsverordnung des Gesetzes Nr. 4 von 1981 über die Schaffung einer Verwaltung der Volksrechtsanwaltschaft. In: Al-garida ar-rasmiya. Tarabulus 5.5.1982, Nr. 14, S. 483-486.

31 Beschluß in: Al-garida ar-rasmiya. Tarabulus 31.1.1983, Nr. 3, S. 126-137. 
mung dieses Komitees den Vorsitzenden (ra'is), die Stellvertreter (wukala') sowie die Inspektoren (mufattisun) der "Verwaltung für die Gerichtsaufsicht". Die insgesamt 26 Mitglieder der Verwaltung 32 haben laut Art. 2 des Beschlusses Nr. 568 von 1982 die Aufgabe, die "Mitarbeiter des Gerichtswesens und der Staatsanwaltschaft" bis zur Stufe der Grundgerichte zu kontrollieren, Klagen gegen sie nachzugehen und die Arbeit der Gerichte zu überprüfen. Úber jedes Mitglied der Gerichte und der Staatsanwaltschaft ist eine persönliche und fachliche Akte anzufertigen (Art. 21). Bei Klagen gegen Vorsitzende, Stellvertreter und Räte der Appellationsgerichte bzw. Vorsitzende der Staatsanwaltschaft beauftragt der Sekretär des Allgemeinen Volkskomitees für Justiz einen der Vorsitzenden der Appellationsgerichte mit der Untersuchung des Sachverhalts (Art. 27). Auf diese Weise soll ein Mißbrauch der richterlichen Gewalt im Interesse eines einzelnen oder einer Gruppe verhindert und der Nimbus der Unbestechlichkeit bei judikativen Entscheidungen gewahrt bleiben. Da die Modalitäten der libyschen Rechtsprechung a priori Systemkonformität sichern, erhält das Gremium der Gerichtsaufsicht die Funktion eines Binde- und Uberwachungsgliedes zwischen zentraler Orientierung und konkreter Realisierung.

Offentliche (und staatliche) Einrichtungen, Gesellschaften und Gremien Libyens vertritt in juristischen Angelegenheiten die "Staatliche Verwaltung für Rechtssachen" (idara qadaya al-hukuma). 33 Das Gesetz Nr. 87 von 1971 wurde im Laufe der Zeit zum einen durch einen Beschluß ergänzt, der diese Verwaltung ermächtigt, die juristischen Interessen libyscher Gesellschaften und Organe im Ausland wahrzunehmen 34, und zum anderen durch eine Reihe von Beschlüssen präzisiert, wonach bestimmte - namentlich erwähnte - Gesellschaften auch im Inland juristisch von der "Verwaltung für Rechtssachen" betreut werden. 35

In Art. 15 des Gesetzes Nr. 51 von 1976 ist die Rolle des ma'dun als Repräsentant der staatlichen Gerichte in Angelegenheiten der Eheschließung und -scheidung festgeschrieben. Diese wichtige traditionell-islamische Funktion, die auch in anderen islamischen Ländem z.T. gesetzlich verankert ist, wurde mit dem "Beschluß des Verwaltungskomitees des Allgemeinen Gremiums für Justizangelegenheiten Nr. 1 von 1987 über das Statut der ma'dunin"36 sanktioniert und in das System der libyschen Justiz eingegliedert.

32 Vgl. die namentliche Aufstellung im "Beschluß des Sekretärs des Allgemeinen Volkskomitees für Justiz Nr. 42 von 1984 über die Reorganisation der Verwaltung für die Gerichtsaufsicht". In: Algarida ar-rasmiya. Tarabulus 7.3.1984, Nr. 6, S. 272-276.

33 Der Name wurde im Zuge der Formienung der "Volksmacht" in "Verwaltung für Rechtssachen" (idara al-qadaya) umgewandelt. Vgl. Gira, A. al-M.A al-A., At-tanzim..., a.a.O., S. 165-168.

34 "Beschluß des Allgemeinen Volkskomitees Nr. 372 von 1982 über die Organisation der Abwicklung von Streitfällen im Ausland". In: Al-garida ar-rasmiya. Tarabulus 6.12.1982, Nr. 37, S. 13121314.

35 Z.B. "Beschluß des Sekretärs des Allgemeinen Volkskomitees für Justiz Nr. 459 von 1983 über die Bevollmächtigung der Verwaltung für Rechtssachen zur Vertretung der 'Gesellschaft von Dima für Urbarmachung und Bau'". In: Al-garida ar-rasmiya. Tarabulus 12.11.1983, Nr. 34, S. 1003. Al-garida ar-rasmiya. Tarabulus 12.4 .1987$, Nr. 10, S. 253-262. 
Struktur und Mechanismus des libyschen Gerichtssystems werden einerseits durch die Militärgerichtsbarkeit ergänzt, die jedoch hier nicht vorgestellt werden soll, und andererseits durch Institutionen und Praktiken modifiziert, die in starkem Maße von politischen Entscheidungen der Führung des Landes abhängig sind. Úber Urteile der Basisvolkskongresse wurde bereits referiert. Am 9.3.1988 beschloß der Allgemeine Volkskongreß die Auflösung der sog. Revolutionsgerichte und die Bildung eines "Volksgerichts" (mahkama as-sab). 37 Künftig solle es - so erklärte M. al-Qaddafi - keine Verfahren vor Sondertribunalen mehr geben. Das "Gesetz Nr. 5 von 1988 über die Schaffung des Volksgerichts"38 regelt in Art. 9, daß sich das "Volksgericht" mit wichtigen "sicherheitspolitischen" Fragen befassen kann. Es ist auch für Schadenersatzklagen gegen Revolutionskomitees zuständig. Trotz einer gewissen Zurückhaltung der libyschen Führungsgruppe um M. al-Qaddafi in bezug auf willkürliche Eingriffe in das Justizsystem seit $1988^{39}$ und bestimunter, auch im Ausland registrierter Maßnahmen (z.B. Ankündigung des Verzichts auf die Todesstrafe) kann von der staatlich postulierten Unabhängigkeit des Gerichtswesens in Libyen nur sehr eingeschränkt gesprochen werden.

Was den Umfang der von den libyschen Gerichten zu behandelnden Fälle angeht, so liegen kaum exakte Angaben vor. Das bezieht sich sowohl auf die Zahl als auch auf den Gegenstand von Gerichtsverfahren. Es finden sich lediglich einige veröffentlichte Anhaltspunkte. In der Zeitung "Al-mizan" erschien eine Aufstellung über Delikte (Verbrechen, Vergehen, Verfehlungen) in den Monaten September/Oktober 1983, die mit 2715 bzw. 2301 angegeben wurde. Die weitaus meisten dieser Straftaten entfielen auf Vergehen $(2126 ; 115$ Verbrechen, 424 Verfehlungen). Dabei wurden 3501 Personen angeklagt, davon lediglich 675 weibliche. In den beiden Monaten 1983 betrug der Wert der geraubten Gegenstände 634.019,670 LD (Libysche Dinar), davon konnten 264.045,310 LD sichergestellt werden (ca. $42 \%$ ) 40 Bei einer Hochrechnung ergeben sich folgende Zahlen: Im Jahre 1983 wurden ca. 30.000 Straftaten verübt, ca. 21.000 Bürger angeklagt und ca. 3,8 Mill. LD entwendet. Bei einer geschätzten Einwohnerzahl von 3,5 Mill. im Jahre 198341 war immerhin jeder 170. Bürger in ein Verbrechen, ein Vergehen oder eine Verfehlung verwickelt, wobei diese Angaben den hohen Anteil von Ausländern in Libyen unberücksichtigt lassen.

37 Az-zahf al-ahdar. Tarabulus 14.3.1988, S. 8.

38 Al-garida ar-rasmiya. Tarabulus 1.6.1988, Sondemummer, S. 1-9.

39 Vgl. Mattes, H., Libyen, in: Nahost Jahrbuch 1989. Politik, Wirtschaft und Gesellschaft in Nordafrika und dem Nahen und Mittleren Osten. Herausgeber: Deutsches Orient-Institut. Th. Koszinowski / H. Mattes, Opladen 1990, S. 111.

40 Die Angaben beziehen sich auf das gesamte Territorium des Landes mit Ausnahme der (damaligen) Baladiyas Murzuq, Gadamis, ubari und Tubruq. Al-Barki, M. A. S., Darura at-tasaddi li-zahira al-garima. In: Al-mizan, Tarabulus 18.3.1984, S. 8.

41 Im Jahre 1984 wurde in Libyen eine Volks- und Gebäudezählung durchgeführt, die vierte nach 1954, 1964 und 1973 und umfassendste bisher. Vgl. dazu az-Zaruq, F., Haula at-tadad al-amm li-ssukan. In: Al-muwazzaf, Tarabulus 5.7.1984, S. 7. 
Ungeachtet der vielfältigen organisatorischen, konzeptionellen und subjektiven Mängel im Wirken des libyschen Justizapparates kristallisiert sich eine Doppelstrategie heraus: Der Prozeß des Aufbaus bzw. der Reorganisation des Gerichtssystems verläuft nicht im generellen Widerspruch zu traditionellen Rechtspraktiken und Rechtsinstitutionen, sondem soll islamische Gepflogenheiten und westliche Erfahrungen zu einem effizienten Ganzen koppeln. Traditionen und Religion müßten - so die offizielle libysche Interpretation - mit der Durchsetzung von Gleichheit und Gerechtigkeit einhergehen. Daß dabei auch in Zukunft Reibungsflächen entstehen, ist angesichts der politischen, sozialen, kulturellen und nicht zuletzt rechtlichen Voraussetzungen nur allzu verständlich. 
state only because of Great Britain's colonial politics, and that Kuwait has always been an integral part of Iraq. Therefore, this article discusses the historical and legal aspects which were brought forward by Saddam Hussein in order to legitimize the invasion and the attempt to incorporate Kuwait into Iraq. Three questions are crucial in this respect:

1. Was Kuwait an integral part of the Ottoman province of Basra?

2. Are there any legitimate claims of Iraq, as a successor state of the Ottoman Empire, to Kuwait?

3. How has Iraq sustained its claims in the past?

The answers to these questions show that Iraqi claims to Kuwait are unfounded.

\section{On the Court System in Libya}

\section{By Hans-Georg Ebert}

As the legal system of Libya is often analysed on the occasion of spectacular or doubtful practices in the judiciary, often with a political background, the institutional structure of the court system itself and its legal basis is seldomly described on a plain legal basis. The article describes the institutional structure in which the courts, attomeys-at-law and court administration work. The article tries to concentrate on the valid statutes and to give an overview, although frequent changes and the dichotomy of state law and sharia make a brief and clear description either impossible or incomplete.

The author also introduces the valid principles governing the legal procedure at court and explains the extent to which the govemment did and does interfere - disrespecting the independence of the judiciary. He concludes that the present Libyan court system is the result of a dual strategy, an attempt to reorganize the legal system integrating the traditional Islamic legal practices and institutions.

\section{Selected Issues Concerning the Operation of Inter-American System for the Protec- tion of Human Rights}

\section{By Antônio Augusto Cançado Trindade}

In the operation of the inter-American system of human rights protection, two issues deserve special attention: the exhaustion of local remedies as a condition of admissibility of 